\title{
Test-Retest Reliability of the Microvascular Oxygenation Recovery Response Subsequent to Submaximal Cycling Exercise
}

Original Research

Trent E. Cayot ${ }^{1}$, Alicia M. Otto ${ }^{1}$, Alex Sikora ${ }^{2}$, Alexandria C. Frick ${ }^{1}$, Nathanial R. Eckert ${ }^{1}$, Stacey L. Gaven ${ }^{2}$

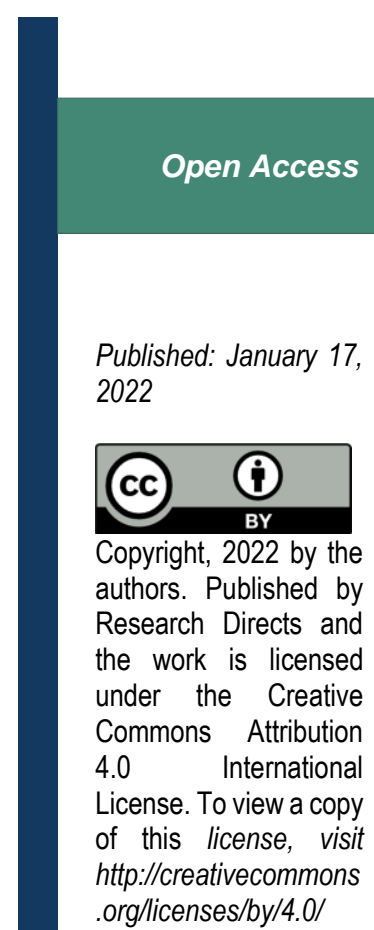

Research Directs in Health Sciences: 2022, Volume 2 (Issue 1): 3

ISSN: 2768-492X
${ }^{1}$ University of Indianapolis, Department of Kinesiology, Health, and Sport Sciences, Indianapolis, Indiana/USA

${ }^{2}$ University of Indianapolis, Department of Atbletic Training, Indianapolis, Indiana/USA

\begin{abstract}
Introduction: The purpose was to quantify the within-session and between-session reliability of halftime (HT) and monoexponential curve fitting (EXP) analyses, when assessing the microvascular tissue oxygenation $\left(\mathrm{StO}_{2}\right)$ recovery response via nearinfrared spectroscopy (NIRS).

Methods: Seventeen subjects completed a submaximal cycling test and 6-minute cool-down on three occasions. The protocol was completed twice during session 1 and once during session 2. $\mathrm{StO}_{2}$ were collected via NIRS from a randomized vastus lateralis. $\mathrm{StO}_{2}$ response from the last minute of exercise and the entire cool-down was analyzed using HT and EXP. Within-session and between-session reliability were examined by mixed, absolute agreement intraclass correlation coefficients (ICC) and standard error of the measurement (SEM).

Results: HT resulted in higher within-session reliability compared to EXP for exercising $\mathrm{StO}_{2}\left(\mathrm{ICC}_{\mathrm{HT}}=0.920, \mathrm{ICC}_{\mathrm{EXP}}=0.865, \mathrm{SEM}_{\mathrm{HT}}=4.9 \Delta \mathrm{BSL}, \mathrm{SEM}_{\mathrm{EXP}}=6.2\right.$ $\Delta \mathrm{BSL})$ and $\mathrm{StO}_{2}$ recovery time $\left(\mathrm{ICC}_{\mathrm{HT}}=0.772, \mathrm{ICC}_{\mathrm{EXP}}=0.720, \mathrm{SEM}_{\mathrm{HT}}=7 \mathrm{sec}\right.$, $\mathrm{SEM}_{\mathrm{EXP}}=9 \mathrm{sec}$ ). Similar between-session reliability for exercising $\mathrm{StO}_{2}$ was observed $\left(\mathrm{ICC}_{\mathrm{HT}}=0.895, \mathrm{ICC}_{\mathrm{EXP}}=0.879, \mathrm{SEM}_{\mathrm{HT}}=5.2 \Delta \mathrm{BSL}, \mathrm{SEM}_{\mathrm{EXP}}=5.4 \Delta \mathrm{BSL}\right)$, however HT elicited higher between-session reliability for $\mathrm{StO}_{2}$ recovery time $\left(\mathrm{ICC}_{\mathrm{HT}}=0.583\right.$, $\mathrm{ICC}_{\mathrm{EXP}}=-0.211, \mathrm{SEM}_{\mathrm{HT}}=7 \mathrm{sec}, \mathrm{SEM}_{\mathrm{EXP}}=15 \mathrm{sec}$ ).

Conclusions: Due to the better within-session (exercising $\mathrm{StO}_{2}, \mathrm{StO}_{2}$ recovery time) and between-session $\left(\mathrm{StO}_{2}\right.$ recovery time) reliability, practitioners are encouraged to use $\mathrm{HT}$ when assessing exercising $\mathrm{StO}_{2}$ and $\mathrm{StO}_{2}$ recovery time.
\end{abstract}

Key Words: Near-Infrared Spectroscopy, Performance Assessment, Curve Fitting Analysis

Corresponding author: Trent E. Cayot, cayott@uindy.edu

\section{Introduction}

Performance of high intensity exercise repeatedly in an intermittent fashion is an important physical characteristic for many athletic events and a characteristic that practitioners might be interested in monitoring throughout a training or rehabilitation intervention. The phosphagen system is heavily relied upon during high intensity, short duration exercise and uses phosphocreatine (PCr) to help meet the increased adenosine triphosphate (ATP) demand. Therefore, to 
sustain performance during subsequent exercise bouts $\mathrm{PCr}$ must be resynthesized from creatine during recovery. Since decreased PCr concentrations have been associated with increased mitochondrial respiration, an exercise bout which decreases the $\mathrm{PCr} /$ creatine ratio will promote $\mathrm{PCr}$ resynthesis ${ }^{1}$, potentially aiding in sustained performance during subsequent exercise ${ }^{2}$. More specifically, creatine can be rephosphorylated via a creatine shuttling mechanism within the inner mitochondrial membrane and the resulting $\mathrm{PCr}$ is then transferred to the cytosol via a voltage-dependent anion channel ${ }^{3,4}$. Since the creatine shuttling mechanism is ATP dependent, requiring oxygen uptake, many studies have investigated the microvascular tissue oxygenation $\left(\mathrm{StO}_{2}\right)$ response during (exercising $\left.\mathrm{StO}_{2}\right)$ and immediately following ( $\mathrm{StO}_{2}$ recovery time) an exercise bout using near-infrared spectroscopy (NIRS) ${ }^{2,5-7}$. After cardiorespiratory training, the exercising skeletal muscle can become more economical during submaximal exercise ${ }^{6}$ and the $\mathrm{StO}_{2}$ response can recover faster immediately following exercise ${ }^{2}$. The faster $\mathrm{StO}_{2}$ recovery time has been associated with PCr resynthesis rates 7 , maximal aerobic speed ${ }^{2}$, the ability to sustain high intensity efforts during multiple exercise bouts ${ }^{2}$, and being a non-invasive indicator of skeletal muscle oxidative capacity ${ }^{8}$.

Because $\mathrm{StO}_{2}$ training adaptations may be advantageous for performance ${ }^{2,6-8}$, practitioners may be interested in monitoring the $\mathrm{StO}_{2}$ response throughout a training ${ }^{2,6}$ or rehabilitation intervention. Previous investigations use multiple analysis techniques to quantify the $\mathrm{StO}_{2}$ variables, including halftime $(\mathrm{HT})^{2,5}$ and monoexponential curve fitting (EXP) ${ }^{7,10}$ analyses. Identifying the most reliable $\mathrm{StO}_{2}$ analysis method would be beneficial for practitioners interested in monitoring potential $\mathrm{StO}_{2}$ changes across training or rehabilitation interventions. Therefore, the primary purpose of the present investigation was to examine the within-session and between-session reliability of two $\mathrm{StO}_{2}$ variables when analyzed by HT and EXP. It was hypothesized that both HT and EXP would provide reliable $\mathrm{StO}_{2}$ measurements.

\section{Scientific Methods}

Participants

Seventeen healthy, active subjects (age $=24 \pm 3$ years, height $=1.74 \pm 0.08 \mathrm{~m}$, weight $=72.2 \pm 11.7 \mathrm{~kg}$, thigh skinfold $=11 \pm 5 \mathrm{~mm}$, estimated maximal oxygen uptake $=40.4 \pm 5.8 \mathrm{ml} / \mathrm{kg} / \mathrm{min}$ ) participated in the investigation. Based upon a previous NIRS investigation ${ }^{11}$, a power analysis indicated that nine subjects were needed for the present investigation (power $=0.80$, alpha $=0.05$ ). Subjects were informed of the investigation's purpose, procedure, and possible risks. All subjects completed an informed consent that was approved by the university's Institutional Review Board for Human Subject Research and was in accordance with the Declaration of Helsinki. Any individual who selfreported a history of metabolic, pulmonary, or cardiovascular disease, an orthopedic related injury in the past 12 months, or who were physically inactive were excluded. Physical activity was defined as participating in structured exercise at least 30 minutes per session, three days a week for the last three months ${ }^{12}$.

\section{Protocol}

Subjects reported to the laboratory on two occasions at approximately the same time of day ( \pm 1 hour) separated by 48 hours. Participants were outfitted with a heart rate monitor (Garmin International Inc., Olathe, Kansas, USA) around their chest and a wireless, continuous-wave NIRS sensor (Moxy Muscle Oxygen Monitor, Fortiori Design, LLC, Hutchinson, Minnesota, USA) on a randomized thigh. Prior to the NIRS sensor placement, the thigh was shaved and cleansed with an alcohol preparation pad. The NIRS sensor was placed midway between the anterior superior iliac spine and the proximal patella over the vastus lateralis ${ }^{11}$ and $\mathrm{StO}_{2}$ data were sampled at $2 \mathrm{~Hz}$. The NIRS sensor was covered with a flexible plastic cover to help prevent stray visible light from affecting the NIRS signal and was secured to the thigh with tape and elastic wraps to limit movement artefact.

After five minutes of rest, resting heart rate was recorded and maximal heart rate was estimated (Estimated $\mathrm{HR}_{\mathrm{MAX}}=$ $208-[0.7 \mathrm{x}$ age $])^{12}$. The resting and estimated maximal heart rates were used to calculate $70 \%$ of the subject's heart rate reserve (HRR). Subjects performed the YMCA Submaximal Cycling Test ${ }^{12}$ (bout 1) on an electromagnetic cycle ergometer (Corival, Lode, Groningen, The Netherlands). All subjects cycled at 25 watts during the first three-minute stage (stage 1) and the work rates used for subsequent stages were dependent upon the subject's heart rate at the end of stage $1(<80 \mathrm{bpm}=125$ watts, $80-89 \mathrm{bpm}=100$ watts, $90-100 \mathrm{bpm}=75$ watts, $>100 \mathrm{bpm}=50$ watts $)$. After the initial work rate increase from stage 1 to stage 2 , the work rate was increased by 25 watts for all subsequent stages. Once the exercising heart rate reached $70 \%$ HRR the subject finished the three-minute stage and then actively recovered for six minutes at 25 watts. In order to account for potential effects that NIRS sensor placement across multiple sessions might have, both within-session (two exercise bouts within the same session where the NIRS sensor was not moved; Bout 1 and Bout 2) and between-session (one exercise bout performed during two sessions where the NIRS sensor was removed and then reapplied; Bout 1 and Bout 3) test-retest reliability analyses were performed. 
Therefore, subjects performed the cycling test and six-minute active recovery again 20 minutes later (bout 2) and once more 48 hours later (bout 3).

All raw $\mathrm{StO}_{2}$ data from the last minute of exercise $(-60 \mathrm{sec}$ to $0 \mathrm{sec})$ and the six-minute active recovery $(0 \mathrm{sec}$ to 360 sec) were interpolated and smoothed using a three-second moving average ${ }^{2}$. For EXP (Figure 1), the StO $\mathrm{O}_{2}$ variables were determined by fitting the data to the following monoexponential curve (Sigma Plot 14.0, Systat Software Inc., Chicago, Illinois, USA), similar to previous investigations ${ }^{7,10}$ :

$\mathrm{StO}_{2}(t)=\mathrm{StO}_{2}(\mathrm{BSL})+\mathrm{Amp} \cdot\left(1-\mathrm{e}^{-\mathrm{t} / \mathrm{tau}}\right)$

$\mathrm{StO}_{2}(\mathrm{BSL})$, Amp, and tau represents the exercising $\mathrm{StO}_{2}, \mathrm{StO}_{2}$ recovery amplitude, and StO 2 recovery time, respectively. For HT, a custom macro function was written (Excel, Microsoft, Redmond, Washington, USA) to calculate the $\mathrm{StO}_{2}$ variables (Figure 1). Specifically, the exercising $\mathrm{StO}_{2}$ was calculated as the average of the last minute of exercise $(-60 \mathrm{sec}$ to $0 \mathrm{sec})$. The $\mathrm{StO}_{2}$ recovery time was determined as the time the $\mathrm{StO}_{2}$ response took to recover $50 \%$ between the exercising $\mathrm{StO}_{2}$ and peak $\mathrm{StO}_{2}{ }^{5}$. Regardless of analysis method, all exercising $\mathrm{StO}_{2}$ values were then normalized to the last 30 seconds of the first exercise stage and thus are presented as a change from baseline $(0 \Delta \mathrm{BSL}$ $=25 \mathrm{~W}$ baseline $)^{11}$.

Figure 1. Representative Example of Monoexponential Curve Fitting Analysis and Halftime Analysis
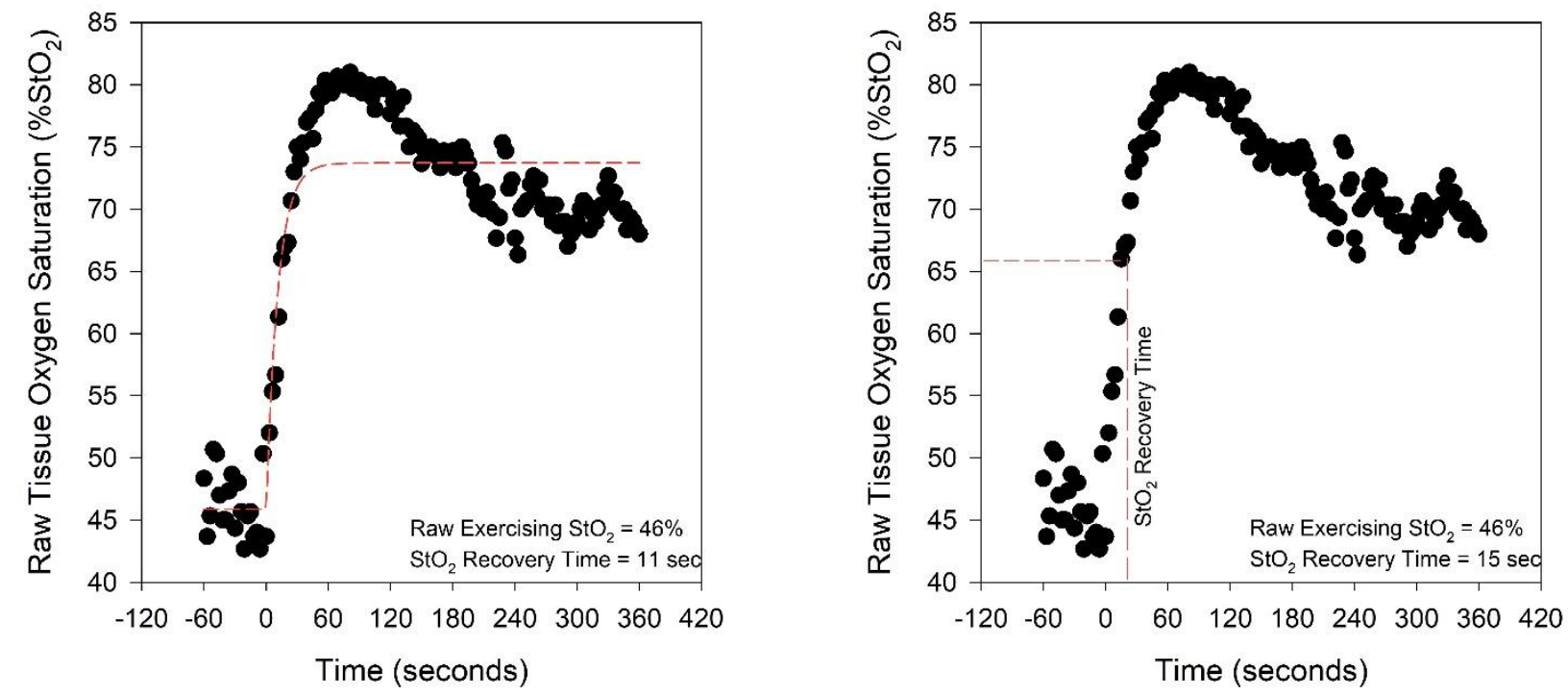

A representative example of the exercising tissue oxygen saturation $\left(\mathrm{StO}_{2}\right)$ response $(-60 \mathrm{sec}$ to $0 \mathrm{sec})$ and tissue oxygen saturation $\left(\mathrm{StO}_{2}\right)$ recovery response $(0 \mathrm{sec}$ to $360 \mathrm{sec})$ analyzed via monoexponential curve fitting analysis (red dashed line in left figure) and halftime analysis (red dashed line in right figure).

\section{Statistical Analysis}

Relative and absolute within-session (bout 1, bout 2) and between-session (bout 1, bout 3) test-retest reliability of the $\mathrm{StO}_{2}$ variables were assess by mixed, absolute agreement intraclass correlation coefficients (ICC) and standard error of the measurement (SEM), respectively. Additionally, the minimal detectable change (MDC) was calculated at a $95 \%$ level of confidence for each $\mathrm{StO}_{2}$ variable. ICC were interpreted as 0.90-1.00 = "excellent", 0.75-0.89 = "good", 0.50$0.74=$ "moderate", and $<0.50=$ "poor". All statistical analyses were performed using Statistical Package for Social Sciences, version 23 (IBM Corporation, Armonk, New York, USA). Statistical significance was set at $\mathrm{p}<0.05$.

\section{Results}

$\mathrm{StO}_{2}$ recovery responses had a moderate-good fit when examined with EXP during bouts 1 ( $\left.\mathrm{r}=0.635-0.997\right)$, 2 $(\mathrm{r}=0.501-0.996)$, and $3(\mathrm{r}=0.791-0.997)$. The within-session and between-session $\mathrm{StO}_{2}$ reliability results are described in Table 1 and 2, respectively. 
Table 1. Within-Session Test-Retest Reliability

\begin{tabular}{|c|c|c|c|c|c|}
\hline Variable & Bout 1 & Bout 2 & ICC & SEM & MDC \\
\hline \multicolumn{6}{|c|}{ Halftime (HT) Analysis } \\
\hline Exercise $\mathrm{StO}_{2}$ & $\begin{array}{c}-13.1 \pm 17.3 \\
\Delta \mathrm{BSL}\end{array}$ & $\begin{array}{c}-16.0 \pm 17.7 \\
\Delta \mathrm{BSL}\end{array}$ & $\begin{array}{c}0.920^{*} \\
0.783-0.971 \\
\text { "Excellent" }\end{array}$ & $4.9 \Delta \mathrm{BSL}$ & 13.6 $\Delta \mathrm{BSL}$ \\
\hline $\mathrm{StO}_{2}$ Recovery Time & $25 \pm 13 \mathrm{sec}$ & $27 \pm 17 \mathrm{sec}$ & $\begin{array}{c}0.772 * \\
0.479-0.911 \\
\text { "Good" }\end{array}$ & $7 \mathrm{sec}$ & $20 \mathrm{sec}$ \\
\hline \multicolumn{6}{|c|}{ Monoexponential Curve Fitting (EXP) Analysis } \\
\hline Exercise $\mathrm{StO}_{2}$ & $\begin{array}{c}-12.4 \pm 16.2 \\
\Delta \mathrm{BSL}\end{array}$ & $\begin{array}{c}-15.6 \pm 17.6 \\
\Delta \mathrm{BSL}\end{array}$ & $\begin{array}{c}0.865 * \\
0.669-0.949 \\
\text { "Good" }\end{array}$ & $6.2 \Delta \mathrm{BSL}$ & $17.1 \Delta \mathrm{BSL}$ \\
\hline $\mathrm{StO}_{2}$ Recovery Time & $24 \pm 18 \mathrm{sec}$ & $24 \pm 16 \mathrm{sec}$ & $\begin{array}{c}0.720^{*} \\
0.373-0.889 \\
\text { "Moderate" }\end{array}$ & $9 \mathrm{sec}$ & $24 \mathrm{sec}$ \\
\hline
\end{tabular}

Group average \pm standard deviation is reported for each variable during Bout 1 and Bout 2 . Intraclass correlations (ICC; single measure results with 95\% confidence intervals and interpretations), standard error of the measurement (SEM), and minimal detectable change (MDC) are provided for each variable. * Significant Intraclass Correlations $(\mathrm{p}<0.05)$.

Table 2. Between-Session Test-Retest Reliability

\begin{tabular}{|c|c|c|c|c|c|}
\hline Variable & Bout 1 & Bout 3 & $\mathrm{ICC}$ & SEM & MDC \\
\hline \multicolumn{6}{|c|}{ Halftime (HT) Analysis } \\
\hline Exercise $\mathrm{StO}_{2}$ & $\begin{array}{c}-13.1 \pm 17.3 \\
\Delta \mathrm{BSL}\end{array}$ & $\begin{array}{c}-17.8 \pm 14.7 \\
\Delta \mathrm{BSL}\end{array}$ & $\begin{array}{c}0.895^{*} \\
0.687-0.963 \\
\text { "Good" }\end{array}$ & $5.2 \Delta \mathrm{BSL}$ & 14.4 $\triangle \mathrm{BSL}$ \\
\hline $\begin{array}{c}\mathrm{StO}_{2} \text { Recovery } \\
\text { Time }\end{array}$ & $25 \pm 13 \mathrm{sec}$ & $21 \pm 9 \mathrm{sec}$ & $\begin{array}{c}0.583 * \\
-0.101-0.846 \\
\text { "Moderate" }\end{array}$ & $7 \mathrm{sec}$ & $21 \mathrm{sec}$ \\
\hline \multicolumn{6}{|c|}{ Monoexponential Curve Fitting (EXP) Analysis } \\
\hline Exercise $\mathrm{StO}_{2}$ & $\begin{array}{c}-12.4 \pm 16.2 \\
\Delta \mathrm{BSL}\end{array}$ & $\begin{array}{c}-17.8 \pm 14.7 \\
\Delta \mathrm{BSL}\end{array}$ & $\begin{array}{c}0.879 * \\
0.614-0.958 \\
\text { "Good" }\end{array}$ & $5.4 \Delta \mathrm{BSL}$ & $14.9 \Delta \mathrm{BSL}$ \\
\hline $\begin{array}{c}\mathrm{StO}_{2} \text { Recovery } \\
\text { Time }\end{array}$ & $24 \pm 18 \mathrm{sec}$ & $17 \pm 7 \mathrm{sec}$ & $\begin{array}{c}-0.211 \\
-1.974-0.541 \\
\text { "Poor" }\end{array}$ & $15 \mathrm{sec}$ & $42 \mathrm{sec}$ \\
\hline
\end{tabular}

Group average \pm standard deviation is reported for each variable during Bout 1 and Bout 3. Intraclass correlations (ICC; average measure results with 95\% confidence intervals and interpretations), standard error of the measurement (SEM), and minimal detectable change (MDC) are provided for each variable. $*$ Significant Intraclass Correlations $(\mathrm{p}<0.05)$.

\section{Discussion}

HT demonstrated better relative (higher ICC) and absolute (lower SEM) within-session reliability for both exercising $\mathrm{StO}_{2}$ and $\mathrm{StO}_{2}$ recovery time compared to EXP (Table 1). Practitioners may be interested that when using HT, they can be confident that a real change occurred if the exercising $\mathrm{StO}_{2}$ or $\mathrm{StO}_{2}$ recovery time changes within a single 
session by $13.6 \Delta \mathrm{BSL}$ or $20 \mathrm{sec}$, respectively. These minimal detectable changes could be used as reference values when interpreting the effects of acute interventions on the $\mathrm{StO}_{2}$ response.

Both HT and EXP provided "good" between-session reliability for exercising $\mathrm{StO}_{2}$ (Table 2). The present exercising $\mathrm{StO}_{2}$ between-session reliability results are similar to previous findings which demonstrated reliable exercising $\mathrm{StO}_{2}$ responses at moderate $(50 \%$ peak work rate, ICC $=0.841)$ and peak $(100 \%$ peak work rate, ICC $=0.873)$ cycling intensities ${ }^{11}$. The "good" between-session reliability for exercising $\mathrm{StO}_{2}$ may allow practitioners to further investigate the efficacy that training ${ }^{6}$ and rehabilitation ${ }^{9}$ interventions have on skeletal muscle exercise economy. According to our results, practitioners using $\mathrm{HT}$ can be confident that a change occurred between sessions when the exercising $\mathrm{StO}_{2}$ response changes by $14.4 \Delta \mathrm{BSL}$.

$\mathrm{HT}$ also resulted in slightly better and statistically significant between-session reliability for $\mathrm{StO}_{2}$ recovery time compared to EXP (Table 2). The lower between-session reliability for $\mathrm{StO}_{2}$ recovery time could have occurred due to differences in NIRS sensor placement between sessions, inter-subject self-selected cycling cadences used, and/or because only one recovery trial was analyzed instead of multiple recovery trials being ensemble averaged and then analyzed ${ }^{10}$. In order to mitigate some potential sources of measurement error, investigators used prominent anatomical landmarks to standardize the NIRS sensor placement ${ }^{11}$. Additionally, an electromagnetic cycle ergometer, which automatically adjusted the resistive torque to maintain constant power output dependent on the cycling cadence, was used to decrease the potential effects that inter-subject, self-selected cycling cadences may have had on the present reliability results. It is important to mention that no differences in exercising $\mathrm{StO}_{2}$ were identified when cycling was performed at different cadences while using the same electromagnetic cycle ergometer ${ }^{13}$. It was a priority of the present investigation to assess testing methods that could easily be implemented in the field or clinical setting. Therefore, instead of having subjects complete multiple exercise and active recovery bouts ${ }^{10}$, only one exercise and active recovery bout was performed. This methodological decision might have led to decreased between-session reliability (Table 2) compared to if multiple exercise and recovery bouts were completed, ensembled averaged, and then analyzed.

The current investigation is not without limitations. NIRS measurements were only recorded from one muscle site and heterogeneous exercising $\mathrm{StO}_{2}$ responses have been reported within the same muscle ${ }^{14}$ and after injury9 . Additionally, since the $\mathrm{StO}_{2}$ response reflects the balance between oxygen delivery and oxygen utilization within the microvasculature, practitioners would not be able to identify the mechanism responsible for any potential change in the $\mathrm{StO}_{2}$ recovery time using the current methodological approach. Suprasystolic occlusion protocols that temporarily occlude local blood flow during the recovery after exercise have been validated in non-invasively assessing mitochondrial function ${ }^{15}$. However, since $\mathrm{StO}_{2}$ recovery time has been shown to be an indicator of muscle oxidative capacity $^{8}$ the current study did not include the suprasystolic occlusion protocol during recovery as practitioners in the field or clinical setting may not have access to the required equipment (rapid cuff inflation system) or the testing population may have contraindications to perform an occlusion protocol. Lastly, cycling exercise was the only exercise modality assessed during the present investigation. The investigators chose to examine cycling exercise due to the low weight bearing properties and increased potential use throughout a clinical/rehabilitation intervention. Therefore, it would be prudent to assess the reliability of $\mathrm{StO}_{2}$ variables using other exercise modalities, such as isometric exercise?

\section{Conclusions}

Based upon the present findings, it is recommended that practitioners interested in non-invasively assessing oxidative capacity after a single exercise bout and active recovery on a cycle ergometer use HT to analyze the exercising $\mathrm{StO}_{2}$ and $\mathrm{StO}_{2}$ recovery time due to the slightly higher within-session and between-session reliability. Practitioners interested in observing if an exercise modality or training/rehabilitation intervention had a real effect on the exercising $\mathrm{StO}_{2}$ response or $\mathrm{StO}_{2}$ recovery time could be confident a change occurred when using the reference values of $\sim 14 \Delta \mathrm{BSL}$ and $\sim 20 \mathrm{sec}$, respectively. Lastly, HT can be analyzed using a commonly available software compared to the more advanced statistical software needed for EXP, possibly making HT accessible to more practitioners within the field and clinical settings.

\section{Acknowledgements}

The authors would like to sincerely thank the participants for volunteering their time and efforts during the research investigation. This study was funded by the University of Indianapolis InQuery Collaborative Research Grant. The authors declare no conflicts of interest. 


\section{References}

1. Walsh B, Tonkonogi M, Soderlund K, Hultman E, Saks V, Sahlin K. The role of phosphorylcreatine and creatine in the regulation of mitochondrial respiration in human skeletal muscle. J Physiol. 2001;537:971-978.

2. Buchheit M, Cormie P, Abbiss C, Ahmaidi S, Nosaka K, Laursen P. Muscle deoxygenation during repeated sprint running: effect of active vs. passive recovery. Int J Sports Med. 2009;30:418-425.

3. Guzun R, Gonzalez-Granillo M, Karu-Varikmaa M, et al. Regulation of respiration in muscle cells in vivo by VDAC through interaction with the cytoskeleton and MtCK within mitochondrial interactosome. Biochim Biophys Acta. 2012;1818:1545-1554.

4. Perry C, Kane D, Herbst E, et al. Mitochondrial creatine kinase activity and phosphate shuttling are acutely regulated by exercise in human skeletal muscle. J Physiol. 2012;590:5475-5486.

5. Chance B, Dait M, Zhang C, Hamaoka T, Hagerman F. Recovery from exercise-induced desaturation in the quadriceps muscles of elite competitive rowers. Am J Physiol. 1992;262:C766-C775.

6. Costes F, Prieur F, Feasson L, Geyssant A, Barthelemy J, Denis C. Influence of training on NIRS muscle oxygen saturation during submaximal exercise. Med Sci Sports Exerc. 2001;33:1484-1489.

7. McCully K, Iotti S, Kendrick K, et al. Simultaneous in vivo measurements of $\mathrm{HbO} 2$ saturation and $\mathrm{PCr}$ kinetics after exercise in normal humans. J Appl Physiol. 1994;77:5-10.

8. Hamaoka T, McCully K, Niwayama M, Chance B. The use of muscle near-infrared spectroscopy in sport, health and medical sciences: recent developments. Philos Trans A Math Phys Eng Sci. 2011;369:4591-4604.

9. Jaffri A, Lynch M, Saliba S, Hart J. Quadriceps oxygenation during exercise in patients with anterior cruciate ligament reconstruction. J Athl Train. 2021; Ahead of Print:1-7.

10. Davies R, Eston R, Poole D, et al. Effect of eccentric exercise-induced muscle damage on the dynamics of muscle oxygenation and pulmonary oxygen uptake. J Appl Physiol. 2008;105:1413-1421.

11. Cayot $\mathrm{T}$, Robinson $\mathrm{S}$, Davis $\mathrm{L}$, et al. Estimating the lactate threshold using wireless near-infrared spectroscopy and threshold detection analyses. Int J Exerc Sci. 2021;14:284-294.

12. Riebe D, Ehrman J, Liguori G, Magal M, eds. ACSM's Guidelines for Exercise Testing and Prescription. 10 ed. Philadelphia, PA: Wolters Kluwer; 2018.

13. Shastri L, Alkhalil M, Forbes C, et al. Skeletal muscle oxygenation during cycling at different power output and cadence. Physiol Rep. 2019;7:e13963.

14. Miyamoto N, Wakahara T, Ema R, Kawakami Y. Non-uniform muscle oxygenation despite uniform neuromuscular activity within the vastus lateralis during fatiguing heavy resistance exercise. Clin Physiol Funct Imaging. 2013;33:463-469.

15. Ryan T, Southern W, Reynolds M, McCully K. A cross-validation of near-infrared spectroscopy measurements of skeletal muscle oxidative capacity with phosphorus magnetic resonance spectroscopy. $J$ Appl Physiol. 2013;115:1757-1766. 


\begin{abstract}
Appendix in 3 second bins (see Protocol section).

Sub NIRS_Recovery0

Range("I2").Value = Application.WorksheetFunction.Average(Range("C2", "C22"))

Range("I5").Value = Application.WorksheetFunction.Max (Range("C22:C450"))

Range("I6").Value = Application.WorksheetFunction.Sum(Range("I5"), -Range("I2"))

Range("I7").Value = Application.WorksheetFunction.Product(Range("I6") / "2")

Range("I10").Value = Application.WorksheetFunction.Sum(Range("I2"), Range("I7"))
\end{abstract}

A custom macro was used in Visual Basic for Applications (VBA), which is included in Microsoft Excel (Redmond, Washington, USA), to quantify the halftime (HT) of the near-infrared spectroscopy (NIRS) recovery response. For this macro function the time data and the microvascular tissue oxygenation $\left(\mathrm{StO}_{2}\right)$ data were placed in column $\mathrm{B}$ and column C, respectively. The "NIRS_Recovery" macro (see below) was then copied and pasted into the VBA dialog box to "run" the macro. The macro calculated the average end exercise $\mathrm{StO}_{2}$ (cell I2), the peak $\mathrm{StO}_{2}$ during recovery (cell I5), the $\mathrm{StO}_{2}$ recovery amplitude (peak $\mathrm{StO}_{2}$ - average end exercise $\mathrm{StO}_{2}$; cell I6), and the $50 \% \mathrm{StO}_{2}$ amplitude (cell I10). The conditional formatting function on the "Home" tab in Excel was then used to identify when the $\mathrm{StO}_{2}$ response (column C) was equal to or above the $50 \% \mathrm{StO} 2$ amplitude (cell I10) and the corresponding time value (from column B) was recorded as the HT. It is important to mention that the first two lines of the custom written macro is dependent upon the sampling frequency in which the NIRS data was recorded and therefore might need to be adjusted based upon the data collection and data analysis procedures. The current macro below is written for NIRS data that is

End Sub 